\title{
Parallel profiling of DNA methylation and hydroxymethylation highlights neuropathology-associated epigenetic variation in Alzheimer's disease
}

Adam R. Smith', Rebecca G. Smith', Ehsan Pishva ${ }^{1,2}$, Eilis Hannon ${ }^{1}$, Janou A. Y. Roubroeks ${ }^{1,2}$, Joe Burrage ${ }^{1}$, Claire Troakes ${ }^{3}$, Safa Al-Sarraj ${ }^{3}$, Carolyn Sloan ${ }^{4}$, Jonathan Mill ${ }^{1}$, Daniel L. van den Hove ${ }^{2,5}$ and Katie Lunnon ${ }^{1 *}$ (D)

\begin{abstract}
Background: Alzheimer's disease is a progressive neurodegenerative disorder that is hypothesized to involve epigenetic dysfunction. Previous studies of DNA modifications in Alzheimer's disease have been unable to distinguish between DNA methylation and DNA hydroxymethylation. DNA hydroxymethylation has been shown to be enriched in the human brain, although its role in Alzheimer's disease has not yet been fully explored. Here, we utilize oxidative bisulfite conversion, in conjunction with the Illumina Infinium Human Methylation 450K microarray, to identify neuropathology-associated differential DNA methylation and DNA hydroxymethylation in the entorhinal cortex.
\end{abstract}

Results: We identified one experiment-wide significant differentially methylated position residing in the WNT5B gene. Next, we investigated pathology-associated regions consisting of multiple adjacent loci. We identified one significant differentially hydroxymethylated region consisting of four probes spanning 104 bases in the FBXL16 gene. We also identified two significant differentially methylated regions: one consisting of two probes in a 93 base-pair region in the ANK1 gene and the other consisting of six probes in a 99-base pair region in the ARID5B gene. We also highlighted three regions that show alterations in unmodified cytosine: two probes in a 39-base pair region of ALLC, two probes in a 69-base pair region in JAG2, and the same six probes in ARID5B that were differentially methylated. Finally, we replicated significant ANK1 disease-associated hypermethylation and hypohydroxymethylation patterns across eight CpG sites in an extended 118-base pair region in an independent cohort using oxidative-bisulfite pyrosequencing.

Conclusions: Our study represents the first epigenome-wide association study of both DNA methylation and hydroxymethylation in Alzheimer's disease entorhinal cortex. We demonstrate that previous estimates of DNA hypermethylation in ANK1 in Alzheimer's disease were underestimates as it is confounded by hypohydroxymethylation.

Keywords: Alzheimer's disease (AD), Brain, Ankyrin 1 (ANK1), DNA methylation (5mC), DNA hydroxymethylation $(5 \mathrm{hmC})$, Entorhinal cortex (EC), Epigenetics, Epigenome-wide association study (EWAS), Illumina Infinium Human Methylation 450K microarray (450K array)

\footnotetext{
* Correspondence: k.lunnon@exeter.ac.uk

${ }^{1}$ College of Medicine and Health, University of Exeter Medical School, Exeter

University, RILD Building Level 4, Royal Devon and Exeter Hospital, Barrack

Rd, Exeter EX2 5DW, UK

Full list of author information is available at the end of the article
}

(C) The Author(s). 2019 Open Access This article is distributed under the terms of the Creative Commons Attribution 4.0 International License (http://creativecommons.org/licenses/by/4.0/), which permits unrestricted use, distribution, and reproduction in any medium, provided you give appropriate credit to the original author(s) and the source, provide a link to the Creative Commons license, and indicate if changes were made. The Creative Commons Public Domain Dedication waiver (http://creativecommons.org/publicdomain/zero/1.0/) applies to the data made available in this article, unless otherwise stated. 


\section{Background}

Alzheimer's disease (AD) affects approximately 35 million people worldwide [1]. It is a progressive neurodegenerative disease that leads to neuronal cell loss and results in severe cognitive decline. The characteristic hallmarks of $\mathrm{AD}$ include neurofibrillary tangles (NFTs) of hyperphosphorylated tau and amyloid beta $(A \beta)$ plaques [2]. The amyloid precursor protein (APP) is normally processed by $\alpha$ - and $\gamma$-secretase (non-amyloidogenic pathway), which forms a soluble, non-toxic $A \beta$ fragment called P3. In AD, APP is cleaved by $\beta$ - and $\gamma$-secretase (amyloidogenic pathway) resulting in the formation of a larger $A \beta$ species, which aggregates to form the characteristic plaques associated with the disease. Although Mendelian inheritance of mutations in the APP gene and the PSEN1 and PSEN2 genes, which encode subunits of the $\gamma$-secretase enzyme, have been demonstrated in early-onset familial AD cases, these only account for $\sim 5 \%$ of disease incidence [3]. The majority of $\mathrm{AD}$ cases are sporadic, occur late in life, and have, as yet, no defined etiology, with common single nucleotide polymorphisms (SNPs) accounting for only a third of disease risk [4].

Epigenetic processes mediate the reversible regulation of gene expression, occurring independently of DNA sequence variation and orchestrate a diverse range of important neurobiological processes in the brain. DNA methylation (5-methylcytosine- $5 \mathrm{mC}$ ) is the best characterized and most stable epigenetic modification [5], and recent epigenome-wide association studies (EWAS) have utilized the Illumina Infinium Human Methylation 450K microarray (450K array) to demonstrate robust and reproducible changes in DNA methylation at a number of loci in AD brain [6,7], including the ANK1 gene. Although the focus of these studies has been on changes in DNA methylation in AD, the sodium bisulfite (BS) conversion approaches used cannot distinguish DNA methylation from DNA hydroxymethylation (5-hydroxymethylcytosine-5hmC). $5 \mathrm{hmC}$ has been previously identified at high levels in the developing [8] and adult brain [9], particularly in neurons [10], and as such may represent an important epigenetic mark to profile in the context of neurodegenerative diseases. Furthermore, levels of $5 \mathrm{hmC}$ potentially mask the true abundance of $5 \mathrm{mC}$ at specific loci in the genome, confounding existing EWAS analyses of AD.

Recent studies have demonstrated that oxidative BS (OxBS) treatment enables the detection of $5 \mathrm{hmC}$ as thymine; therefore, by running matched BS- and OxBS-treated samples in parallel, it is possible to generate a quantitative measurement for total DNA modifications (BS data), DNA methylation (OxBS data), and, by proxy, DNA hydroxymethylation (BS data - OxBS data) and unmodified cytosine (uC) levels (1-BS data) [11].
We have previously utilized this method in conjunction with Illumina $450 \mathrm{~K}$ arrays to profile $5 \mathrm{mC}$ and $5 \mathrm{hmC}$ levels in parallel in post-mortem brain tissue from non-demented individuals to demonstrate brain region-specific differences in DNA modifications [11]. Here, we perform an EWAS of $5 \mathrm{mC}, 5 \mathrm{hmC}$, and $\mathrm{uC}$ using the $450 \mathrm{~K}$ array and brain tissue from 96 donors representing the spectrum of $\mathrm{AD}$ pathology defined by Braak staging, a standardized measure of NFT burden determined at autopsy [12], ranging from no AD pathology (Braak 0) to late-stage AD (Braak VI) (Table 1). We looked for an association with Braak stage, rather than clinical diagnosis of disease, as clinical symptoms of the disease are only seen in the middle (limbic) stages of disease (Braak III-IV) [12], while by using Braak stage, we were able to capture epigenetic changes in the earliest stages of disease. From each donor, we analyzed the entorhinal cortex (EC) as this is thought to be the starting point of $\mathrm{AD}$ pathology in the cortex with pathology seen here in Braak stage II [12].

\section{Results and discussion \\ Highly reproducible alterations in total DNA modifications are detectable in AD EC}

Previous studies have used BS DNA in conjunction with the $450 \mathrm{~K}$ array to identify loci that show AD-associated alterations in total DNA modifications [6, 7]. To assess the reproducibility of our results, we first compared the effect size of the 100 top-ranked Braak-associated differentially modified positions identified in our BS data generated in the "discovery cohort," with BS data previously published on an independent set of samples from the EC in AD ("validation cohort 1") [7]. Across these sites, there were significant consistent effects between the two studies (Fig. 1a: sign test $P=2.04 \times 10^{-4}$ ), with the effect sizes highly correlated between studies $(r=0.591, P=$ $\left.8.92 \times 10^{-11}\right)$. There were also significant consistent effects across studies for the 100 top-ranked EC Braak-associated differentially modified positions identified in the previous study [7] (Fig. 1b: sign test $P=$ $2.04 \times 10^{-4}$ ), with the effect sizes highly correlated between studies $\left(r=0.550, P=2.95 \times 10^{-9}\right)$. This suggests that although different BS conversion methods and donors were used, there are consistent and reproducible changes in total DNA modifications in the EC of donors with $\mathrm{AD}$.

\section{Locus-specific changes in DNA methylation and hydroxymethylation occur in AD EC}

We were interested to identify specific loci that showed Braak-associated alterations in levels of $5 \mathrm{mC}, 5 \mathrm{hmC}$, or $\mathrm{uC}$ in the EC (Fig. 2a). We identified one Braak-associated differentially methylated position (DMP) (cg10696062) that was experiment-wide 
Table 1 Sample and demographic information for the three cohorts used

\begin{tabular}{|c|c|c|c|c|c|}
\hline \multirow[b]{2}{*}{ Treatment } & \multicolumn{2}{|c|}{ Discovery cohort (450K array) } & \multirow{2}{*}{$\begin{array}{l}\text { Validation cohort } 1 \text { (450K array) } \\
\text { BS }\end{array}$} & \multicolumn{2}{|c|}{ Validation cohort 2 (pyrosequencing) } \\
\hline & BS & OxBS & & BS & OxBS \\
\hline$n$ passed QC & 91 & 85 & 104 & 96 & 92 \\
\hline Gender $[\mathrm{M} / \mathrm{F}]$ & $51 / 40$ & $48 / 37$ & $42 / 62$ & $54 / 42$ & $52 / 40$ \\
\hline Mean age $( \pm S D)$ & $81.2(9.5)$ & $81.3(9.5)$ & $84.9(8.7)$ & $85.0(7.2)$ & $84.8(7.3)$ \\
\hline \multicolumn{6}{|l|}{ Braak stage } \\
\hline 0 & 8 & 7 & 5 & 5 & 5 \\
\hline । & 3 & 3 & 11 & 6 & 6 \\
\hline$\|$ & 11 & 10 & 8 & 37 & 35 \\
\hline III & 6 & 6 & 13 & 0 & 0 \\
\hline IV & 8 & 7 & 5 & 0 & 0 \\
\hline V & 18 & 17 & 18 & 23 & 22 \\
\hline $\mathrm{Vl}$ & 37 & 35 & 44 & 25 & 24 \\
\hline Mean PMI [min] $( \pm S D)$ & $2539.5(1288.1)$ & $2490.7(1288.5)$ & $1997.6(1227.0)$ & $2960.6(1943.8)$ & $2958.9(1958.7)$ \\
\hline
\end{tabular}

The discovery cohort consisted of 450K array BS and OxBS data generated in 96 individuals from the MRC London Brain Bank for Neurodegenerative Disease, with 91 BS arrays and 85 OxBS arrays passing quality control (QC). Validation cohort 1 consisted of previously published 450K array BS data generated in an independent cohort of 104 individuals also from the MRC London Brain Bank for Neurodegenerative Disease [7]. Validation cohort 2 consisted of pyrosequencing BS and OxBS data we generated in an independent cohort of 96 individuals from the Thomas Willis Oxford Brain Collection, with 96 BS and 92 OxBS samples passing QC. For each cohort, we analyzed the entorhinal cortex. Shown for each dataset are the number of samples ( $n$ ) that passed QC, distribution of sex, mean age, Braak stage spread, and postmortem interval (PMI) ( \pm standard deviation (SD))

significant $\left(P<2.4 \times 10^{-7}\right)$, residing within the first intron of the largest isoform of WNT5B, a gene which is known to have four isoform variants in humans (Additional file 1: Table S1). We observed a $12.95 \%$ change $(\Delta)$ in DNA methylation levels between individuals with Braak 0 and Braak $\mathrm{VI}$, with increased $5 \mathrm{mC}$ seen with increased disease severity $\left(P=1.47 \times 10^{-7}\right)$, with a concurrent nominal decrease in $5 \mathrm{hmC}(\Delta=-9.04 \%, P=1.56 \times$ $\left.10^{-4}\right)$ and $\mathrm{uC}(\Delta=-3.94 \%, P=0.02)$ (Fig. 2b, c). WNT5B is part of the WNT gene family, and disruption of the WNT signaling pathway has been previously implicated in neurodegeneration and AD [13, 14]. Research specifically into a role for WNT5B in AD is limited; one study profiled gene expression for all WNT genes in EC and showed no difference in WNT5B between AD cases and non-demented controls [15]. However, the study was limited to only 10 individuals per group, which highlights the need for further research into this gene. We observed no experiment-wide significant Braak-associated differentially hydroxymethylated positions (DHPs) (Additional file 1: Table S2) or differentially unmodified cytosine positions (DUPs) (Additional file 1: Table S3) in the EC. It is possible that we did not identify any experiment-wide significant DHPs for two reasons. First, we have previously shown that $5 \mathrm{hmC}$ is significantly depleted in the proximal promoter of genes [11], and $40 \%$ of probes on the $450 \mathrm{~K}$ array primarily map to these locations [16]. Second, we used a stringent cutoff for measuring $5 \mathrm{hmC}$ to avoid false positive results, only including samples in the $5 \mathrm{hmC}$ analysis if they had a mean beta value $>0.1$ on both the BS and oxBS $450 \mathrm{~K}$ arrays and if $5 \mathrm{hmC}$ was present in more than half of the sample population, meaning that only 219,435 probes were used for the $5 \mathrm{hmC}$ analysis (see the "Methods" section).

\section{Regional analyses identify adjacent DMPs, DHPs, and DUPs}

Next, we performed an analysis to identify spatially correlated regions consisting of $>2$ neighboring DMPs (differentially methylated regions-DMRs), DHPs (differentially hydroxymethylated regions-DHRs), or DUPs (differentially unmodified regions-DURs) that had a Sidak-corrected $P$ value $<0.05$ within a 500 -bp sliding window (comb-p [17]). We identified two DMRs (Table 2 (A)): a two-probe DMR in the ANK1 gene (Fig. 3a) (Sidak-corrected $P=6.50 \times 10^{-4}$ ) and a six-probe DMR spanning $99 \mathrm{bp}$ in the ARID5B gene (Fig. 3b) (Sidak-corrected $P=1.18 \times 10^{-3}$ ). The two DMPs within the ANK1 DMR have previously been nominated as DMPs in AD cortex [6,7]. ARID5B has not been nominated in previous EWAS and encodes a DNA binding protein, which when associated with phosphorylated PHF2 becomes demethylated, and allows the PHF2-ARID5B complex to function as a histone H3K9Me2 demethylase [18] and increase the target gene's expression. Interestingly, genetic variation in $A R I D 5 B$ has been shown to be associated with $A D$ in two genome-wide association studies (GWAS) $[19,20]$. However, these studies showed opposing effects of the SNP (rs2588969) on AD risk. A study has since genotyped rs2588969 and another SNP from the Hollingworth et al. GWAS study (rs4948288) and demonstrated 

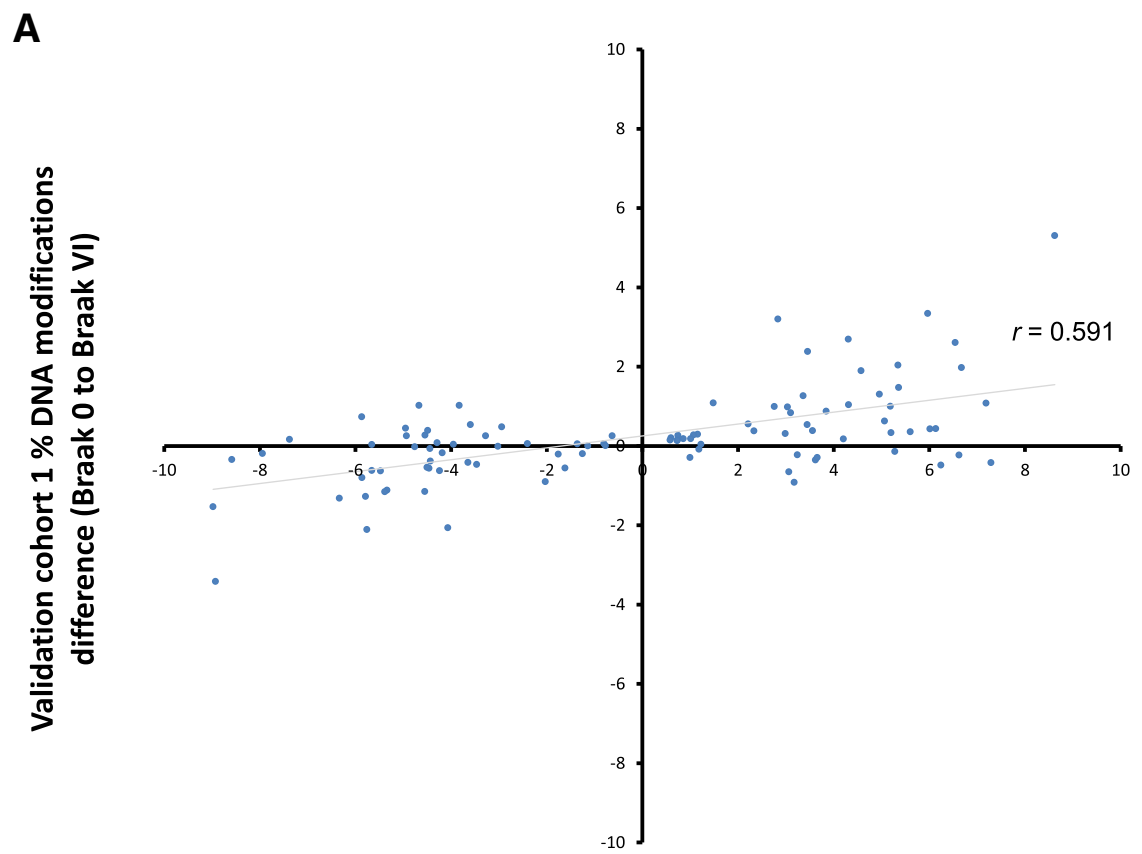

Discovery cohort \% DNA modifications difference (Braak 0 to Braak VI)

B

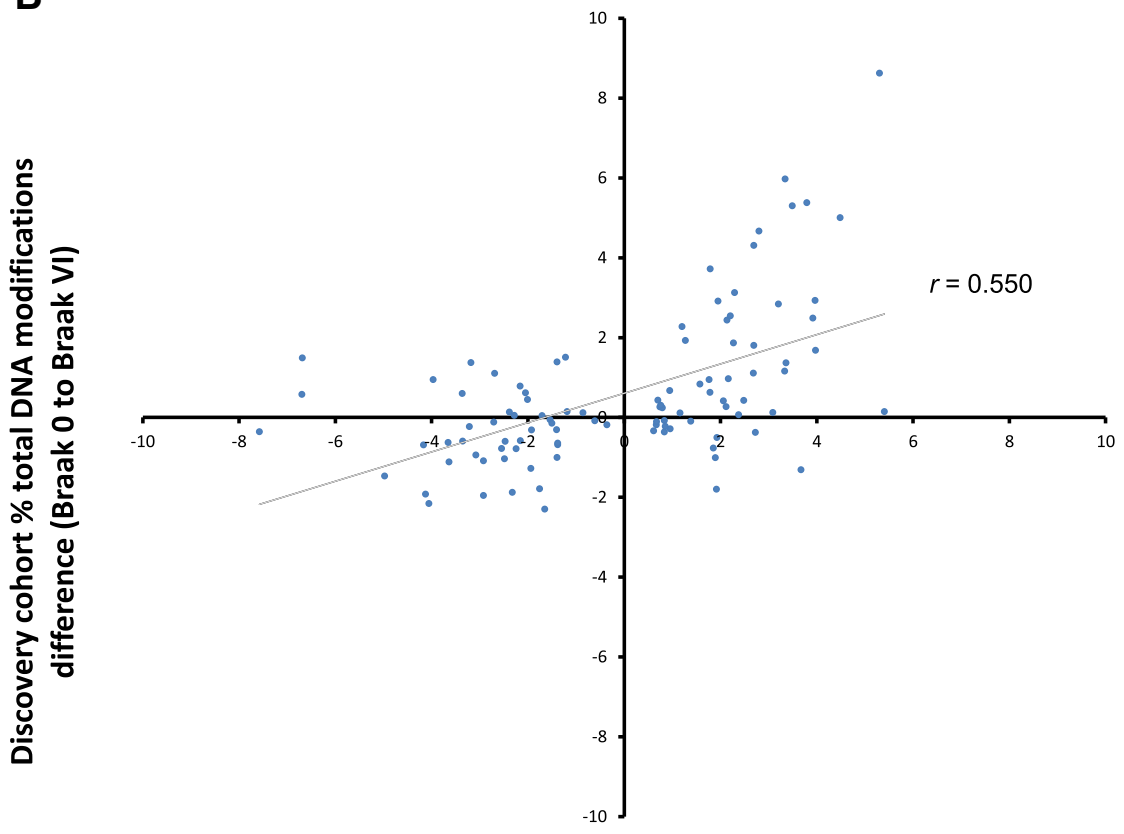

Validation cohort $1 \%$ total DNA modifications difference (Braak 0 to Braak VI)

Fig. 1 Braak-associated differentially modified positions identified in the EC in BS data in this study are consistent with those identified in previous analyses of AD brain. a There was a consistent direction of effect for the top 100 Braak-associated differentially modified positions identified in the EC in BS data in the "discovery cohort" when compared with the same probes in a previously published dataset of Braakassociated differentially modified positions in the EC from BS data ("validation cohort 1") (sign test $P=2.04 \times 10^{-4}$ ), with the effect sizes highly correlated between studies $\left(r=0.591, P=8.92 \times 10^{-11}\right)$. b Similarly, there was a consistent direction of effect for the previously published top 100 Braak-associated differentially modified positions in the EC in BS data ("validation cohort 1") when compared with the same probes in the current study (sign test $\left.P=2.04 \times 10^{-4}\right)$, with the effect sizes highly correlated between studies $\left(r=0.550, P=2.95 \times 10^{-9}\right)$ 


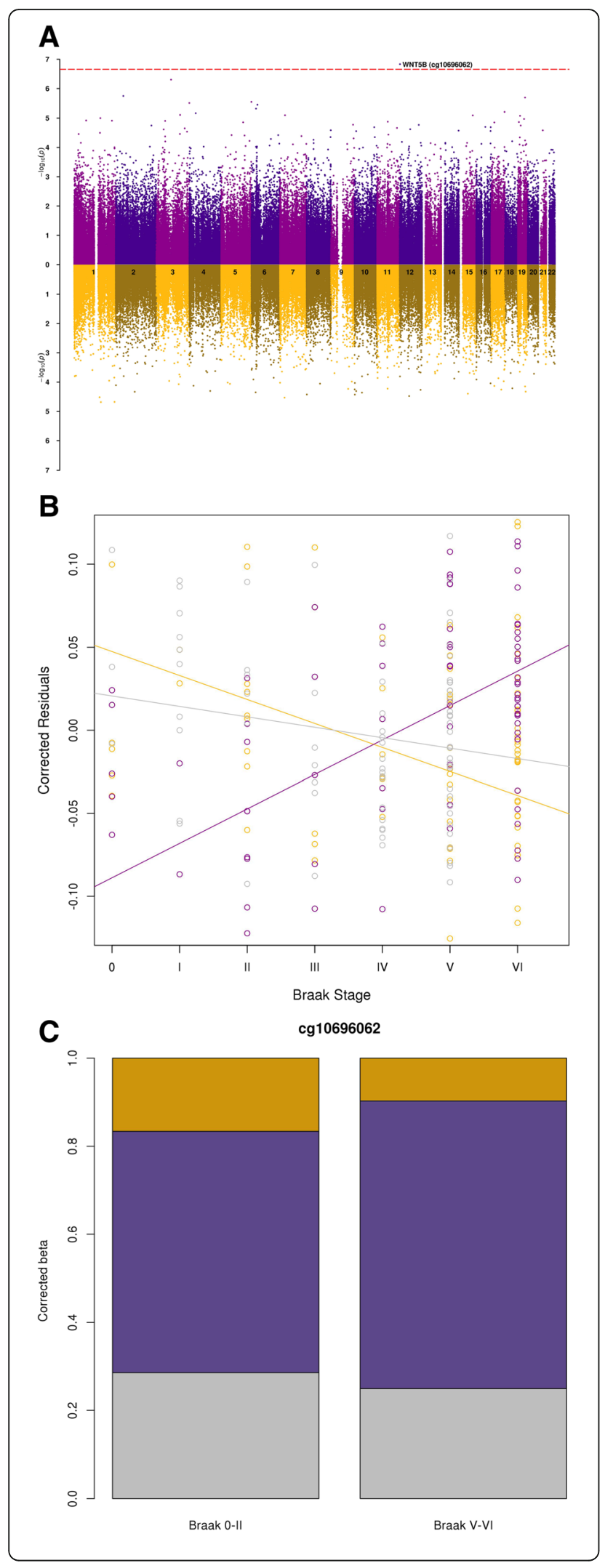

Fig. 2 Alterations in DNA methylation at cg10696062 in WNT5B are associated with AD neuropathology in the EC. a A Manhattan plot of association between DNA methylation (purple, above $X$-axis) and DNA hydroxymethylation (yellow, below $X$-axis) with Braak stage in the EC highlights associations at loci across the genome. One DMP in WNT5B (cg10696062) was experiment-wide significant $(P<2.4 \times$ $\left.10^{-7}\right)$. With increasing Braak stage cg10696062 showed an experiment-wide significant increase in $5 \mathrm{mC}\left(P=1.47 \times 10^{-7}\right)$, in parallel with a nominally significant decrease in $5 \mathrm{hmC}(P=1.56 \times$ $\left.10^{-4}\right)$ and $\mathrm{uC}(P=0.02)$. b Scatter plot of residual values, corrected for age, sex, and neuron/glia proportion, against Braak stage for cg10696062 with purple circles representing $5 \mathrm{mC}$ levels, yellow circles representing $5 \mathrm{hmC}$ levels, and gray circles representing uC levels. Regression lines are shown for $5 \mathrm{mC}$ (purple line), $5 \mathrm{hmC}$ (yellow line), and uC levels (gray line). c Stack chart depicting differences in adjusted beta value (controlling for age, sex, and neuron/glia proportion) between individuals with low (Braak Stage 0-II) and high (Braak Stage V-VI) pathology for $5 \mathrm{mC}$ (purple), $5 \mathrm{hmC}$ (yellow), and UC (gray) levels

a significant association with $\mathrm{AD}$ [21]. However, these effects were lost when the data was adjusted for the co-variates of age at diagnosis, sex, and $A P O E$ \&4 status, which the authors believed could account for the discrepancy between the two GWAS reports. We found one DHR (Table 2 (B)) consisting of four probes in a 104-bp region of the FBXL16 gene (Fig. 3c) (Sidak-corrected $\left.P=1.55 \times 10^{-4}\right)$. FBXL16 is a member of the F-box protein family, and expression of this gene has been shown to be upregulated fourfold in a rat model of Parkinson's disease (PD) [22]. However, a microarray study of acutely isolated microglia from the cortex of an AD mouse model showed a 2.6-fold downregulation in the AD transgenic animals compared to aged wild-type control mice [23]. We identified three DURs (Table 2 (C)): a two-probe DUR in JAG2 (Fig. 3d) (Sidak-corrected $\left.P=1.75 \times 10^{-5}\right)$, a two-probe DUR in $A L L C$ (Fig. 3e) (Sidak-corrected $P=1.61 \times 10^{-3}$ ), and a six-probe DUR in ARID5B (Fig. 3b) (Sidak-corrected $P=$ $2.49 \times 10^{-4}$ ), covering the same 99-bp region where we observed a DMR. JAG2 is a member of the Notch family and can be cleaved by ADAM10, which is the constitutive $\alpha$-secretase involved in the non-amyloidogenic processing of APP [24]. Interestingly, $\mathrm{He}$ et al. also showed that JAG2 is only weakly cleaved by BACE1, which is the $\beta$-secretase enzyme responsible for the amyloidogenic processing of APP.

\section{ANK1 shows differential DNA methylation in AD}

Interestingly, the two DMPs within the ANK1 DMR have previously been nominated as DMPs in AD EC in another EWAS [7]. Although these studies claimed to identify neuropathology-associated hypermethylation at both loci, they were actually unable to discriminate between $5 \mathrm{mC}$ and $5 \mathrm{hmC}$ due to the utilization of BS-treated DNA and, as such, represent a sum of both 
Table 2 Identification of multi-probe regions associated with Braak stage

\begin{tabular}{|c|c|c|c|c|c|}
\hline \multicolumn{6}{|l|}{ A } \\
\hline Position & Gene name & Probes in DMR & Putative region $P$ & Sidak-corrected $P$ & Probes in DMR \\
\hline 8:41519307-41519400 & ANK1 & 2 & $8.51 \mathrm{E}-08$ & $6.50 \mathrm{E}-04$ & $\begin{array}{l}\text { cg05066959 } \\
\text { cg11823178 }\end{array}$ \\
\hline 10:63809072-63809171 & $A R I D 5 B$ & 6 & $1.64 \mathrm{E}-07$ & $1.18 \mathrm{E}-03$ & $\begin{array}{l}\text { cg00928816 } \\
\text { cg07520810 } \\
\text { cg14789659 } \\
\text { cg16389209 } \\
\text { cg16401465 } \\
\text { cg20746552 }\end{array}$ \\
\hline \multicolumn{6}{|l|}{ B } \\
\hline Position & Gene name & Probes in DHR & Putative region $P$ & Sidak-corrected $P$ & Probes in DHR \\
\hline $16: 745662-745766$ & FBXL16 & 4 & $3.75 \mathrm{E}-08$ & $1.55 \mathrm{E}-04$ & $\begin{array}{l}\text { cg01003448 } \\
\text { cg01195246 } \\
\text { cg02958327 } \\
\text { cg07482202 }\end{array}$ \\
\hline \multicolumn{6}{|l|}{ C } \\
\hline Position & Gene name & Probes in DUR & Putative region $P$ & Sidak-corrected $P$ & Probes in DUR \\
\hline 14:105619633-105619702 & $J A G 2$ & 2 & 1.70E-09 & $1.75 \mathrm{E}-05$ & $\begin{array}{l}\text { cg13617301 } \\
\text { cg19893664 }\end{array}$ \\
\hline 10:63809072-63809171 & $A R I D 5 B$ & 6 & $3.46 \mathrm{E}-08$ & $2.49 \mathrm{E}-04$ & $\begin{array}{l}\text { cg00928816 } \\
\text { cg07520810 } \\
\text { cg14789659 } \\
\text { cg16389209 } \\
\text { cg16401465 } \\
\text { cg20746552 }\end{array}$ \\
\hline 2:3714277-3714316 & ALLC & 2 & $8.84 \mathrm{E}-08$ & $1.61 \mathrm{E}-03$ & $\begin{array}{l}\text { cg04307702 } \\
\text { cg27089736 }\end{array}$ \\
\hline
\end{tabular}

Using Comb-p, we identified spatially correlated $P$ values within a 500-bp sliding window [17]. DMRs are shown in (A), DHRs are shown in (B), and DURs are shown in (C). Shown are regions with a Sidak-corrected $P$ value $<0.05$

modifications. In the current study, we demonstrate nominally significant hypermethylation at both loci (Fig. 4a: cg05066959: $\Delta=13.10, \quad P=1.13 \times 10^{-4}$ and Fig. 4b: $\operatorname{cg} 11823178: \Delta=9.39, P=5.48 \times 10^{-5}$ ), in parallel with a nominally significant decrease in $\mathrm{uC}$ (cg11823178: $\Delta=-5.03, P=1.19 \times 10^{-3} ; \operatorname{cg} 05066959: \Delta=-9.06, P=$ $\left.1.18 \times 10^{-4}\right)$, and a nominally significant decrease in $5 \mathrm{hmC}$ at $\operatorname{cg} 11823178(\Delta=-4.36, P=0.02)$, with a non-significant decrease in $5 \mathrm{hmC}$ at $\operatorname{cg} 05066959(\Delta=-$ $4.04, P=0.14)$. These figures highlight how alterations in $5 \mathrm{mC}, 5 \mathrm{hmC}$, and $\mathrm{uC}$ are seen early in the disease process. Our findings indicate that previous studies of DNA methylation in ANK1 have underestimated increments in $5 \mathrm{mC}$ in disease due to confounding by $5 \mathrm{hmC}$ and could suggest the loss of active DNA demethylation of ANK1 in $\mathrm{AD}$, as both $5 \mathrm{hmC}$ and $\mathrm{uC}$ are lower in disease.

\section{Oxidative-bisulfite pyrosequencing validation of ANK1}

To further investigate whether ANK1 epigenetic changes associated with neuropathology involve DNA hypermethylation, and not hyperhydroxymethylation, we used OxBS pyrosequencing to quantify DNA modifications across an extended region of 118 bp spanning eight CpG sites, including cg11823178 and cg05066959. DNA used for this study was from an independent collection of EC tissue $(n=96)$ obtained from the Thomas Willis Oxford Brain Collection ("validation cohort 2") (Table 1) [25]. In this cohort, we did not have access to Braak stage III or IV samples, and as such analyzed data using a case (Braak V-VI)/control (Braak 0-II) analysis model (see the "Methods" section). Of the eight CpG sites assessed, seven were characterized by nominally significant $(P<$ $0.05) \mathrm{AD}$-associated hypermethylation (Fig. 5a). Interestingly, significant $\mathrm{AD}$-associated hypohydroxymethylation was also seen at four of the eight CpG sites (Fig. 5b), although the two loci covered by the $450 \mathrm{~K}$ array showed no significant difference, further confirming our $450 \mathrm{~K}$ array findings. We observed a significant decrease in $\mathrm{uC}$ at four of the eight sites (Fig. 5c). When we averaged $5 \mathrm{mC}, 5 \mathrm{hmC}$, and $\mathrm{uC}$ levels across the eight $\mathrm{CpG}$ sites in the 118-bp region, we saw a significant $6.01 \%$ increase in $5 \mathrm{mC}\left(P=1.23 \times 10^{-5}\right)$, a significant $3.87 \%$ decrease in $\mathrm{uC}$ $\left(P=6.72 \times 10^{-3}\right)$, and a trend towards a $2.09 \%$ decrease in $5 \mathrm{hmC}(P=0.058)$ in individuals with Braak stage $\mathrm{V}-$ VI, compared to Braak stage 0-II (Fig. 5d). This data confirms our array-based results and further illustrates that previous studies of ANK1 DNA modifications in 


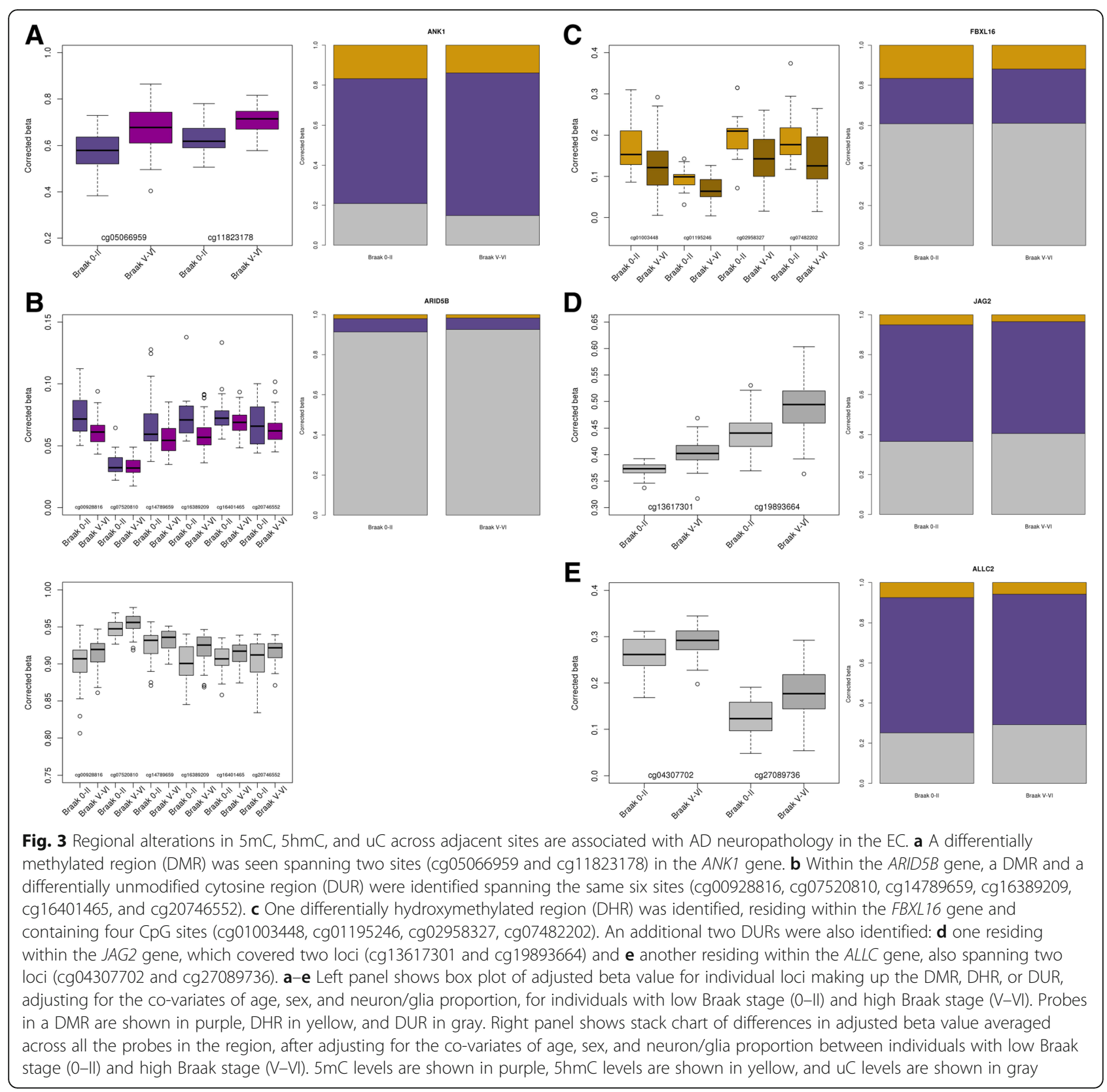

$\mathrm{AD}$ have underestimated disease-associated changes in $5 \mathrm{mC}$.

Several recent studies have reported the links between epigenetic alterations at the ANK1 locus and the development of $\operatorname{AD}[6,7,26]$. A recent study showed that alterations in total DNA modifications across the 118-bp amplicon in $A N K 1$ are seen in the EC in individuals with $\mathrm{AD}$, Huntington's disease (HD), and PD [27]. Interestingly, individuals with dementia with Lewy bodies (DLB) or vascular dementia $(\mathrm{VaD})$ only showed increased DNA modifications in individuals with co-existing AD pathology. The ANK1 protein is a plasma-bound membrane protein that contains an ankyrin repeat domain, which modulates interactions between cytoskeletal and membrane proteins [28]. One of the main functions of ANK1 is compartmentalization and maintenance of the plasma membrane, and it is possible that the altered expression of this gene could lead to cell membrane dysfunction in $\mathrm{AD}$ [7]. Recent studies suggest that alterations in ANK1 in $\mathrm{AD}$ are glial-derived; a recent EWAS was performed on sorted neuronal and glial nuclei from the temporal cortex and frontal cortex of AD and control donors and demonstrated that Braak-associated alterations in ANK1 total DNA modifications are of glial origin [29]. Another study has shown that microglia, the brain's resident macrophages, display a greater than fourfold increase in 


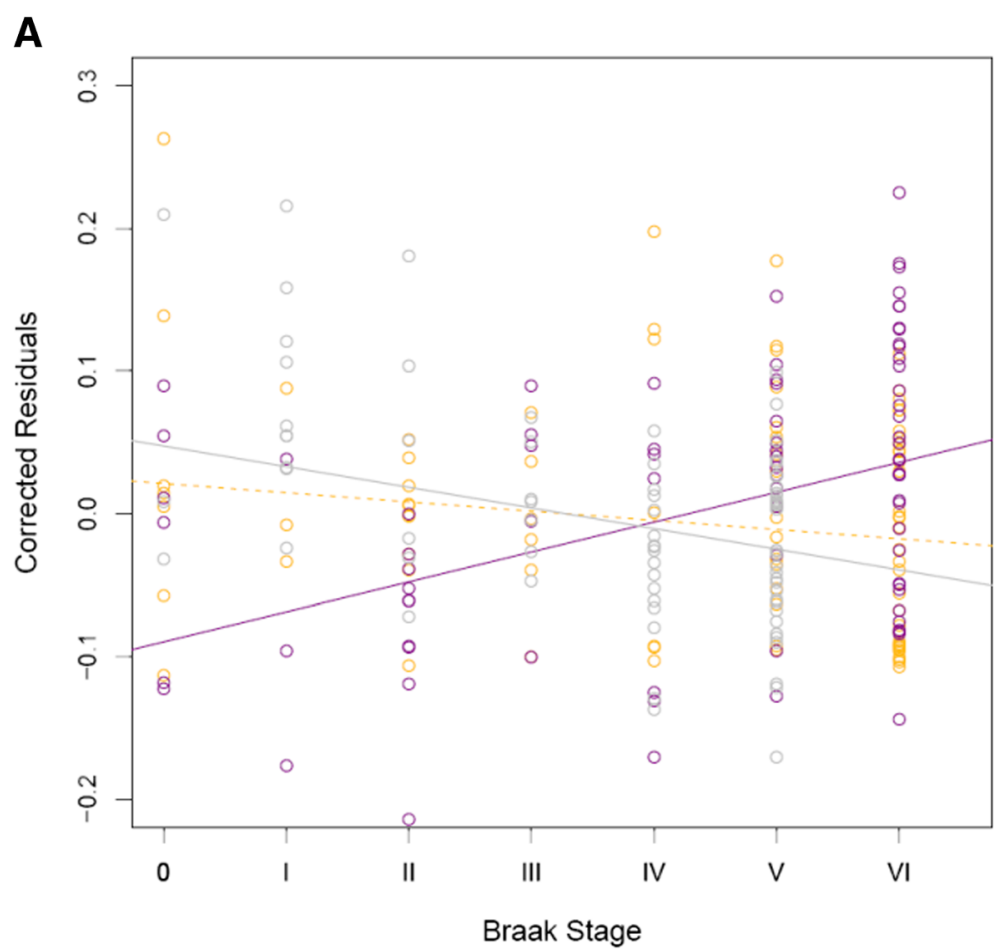

B

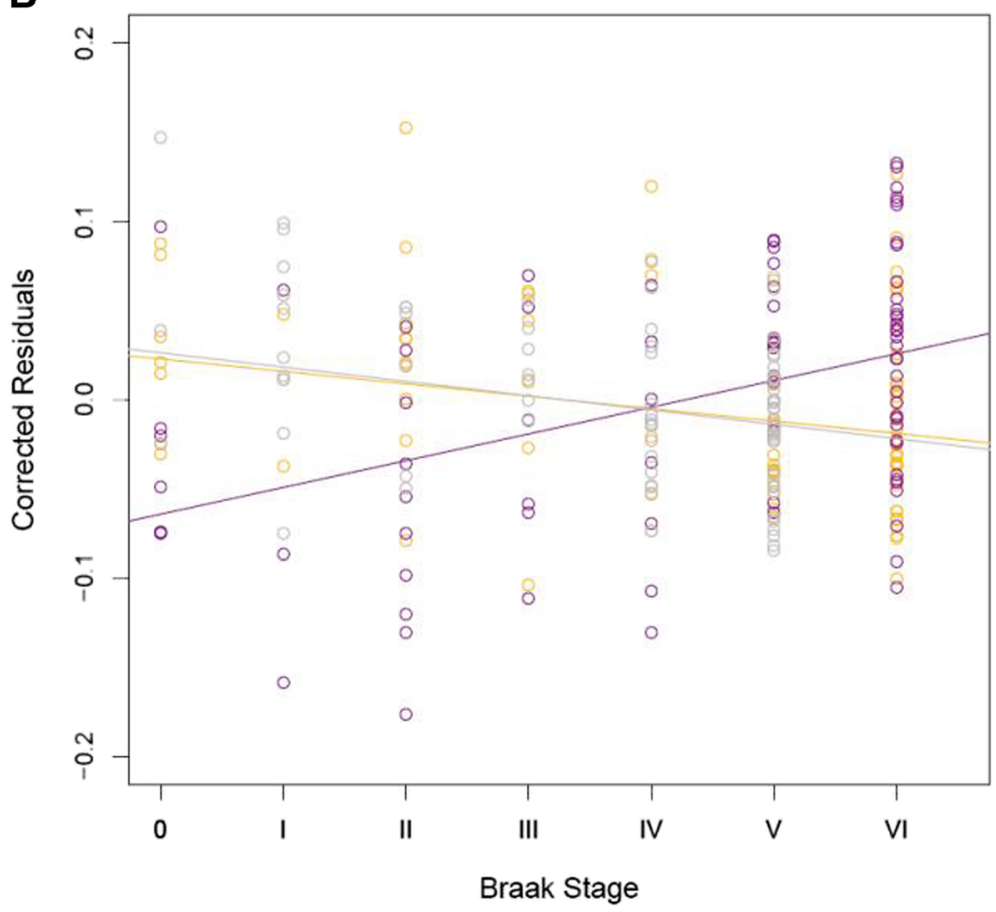

Fig. 4 Two probes within ANK1 are differentially methylated with respect to Braak stage. Shown are beta values for a cg05066959 and $\mathbf{b}$ cg11823178 when adjusted for the co-variates of age, sex, and neuron/glia proportion (Y-axis), against Braak stage (X-axis). Purple circles represent $5 \mathrm{mC}$ levels, yellow circles represent $5 \mathrm{hmC}$ levels, and gray circles represent $\mathrm{uC}$ levels. Regression lines are shown for $5 \mathrm{mC}$ (purple line), $5 \mathrm{hmC}$ (yellow line), and $\mathrm{uC}$ (gray line) levels. Solid regression lines indicate $P<0.05$, while dashed lines indicate $P>0.05$

ANK1 expression in AD compared to control brain samples [26], suggesting it is possible that ANK1 DNA methylation levels will have a downstream effect on immune and inflammatory regulation in the brain. Although DNA methylation in the promoter regions of genes is traditionally thought to bring about gene 

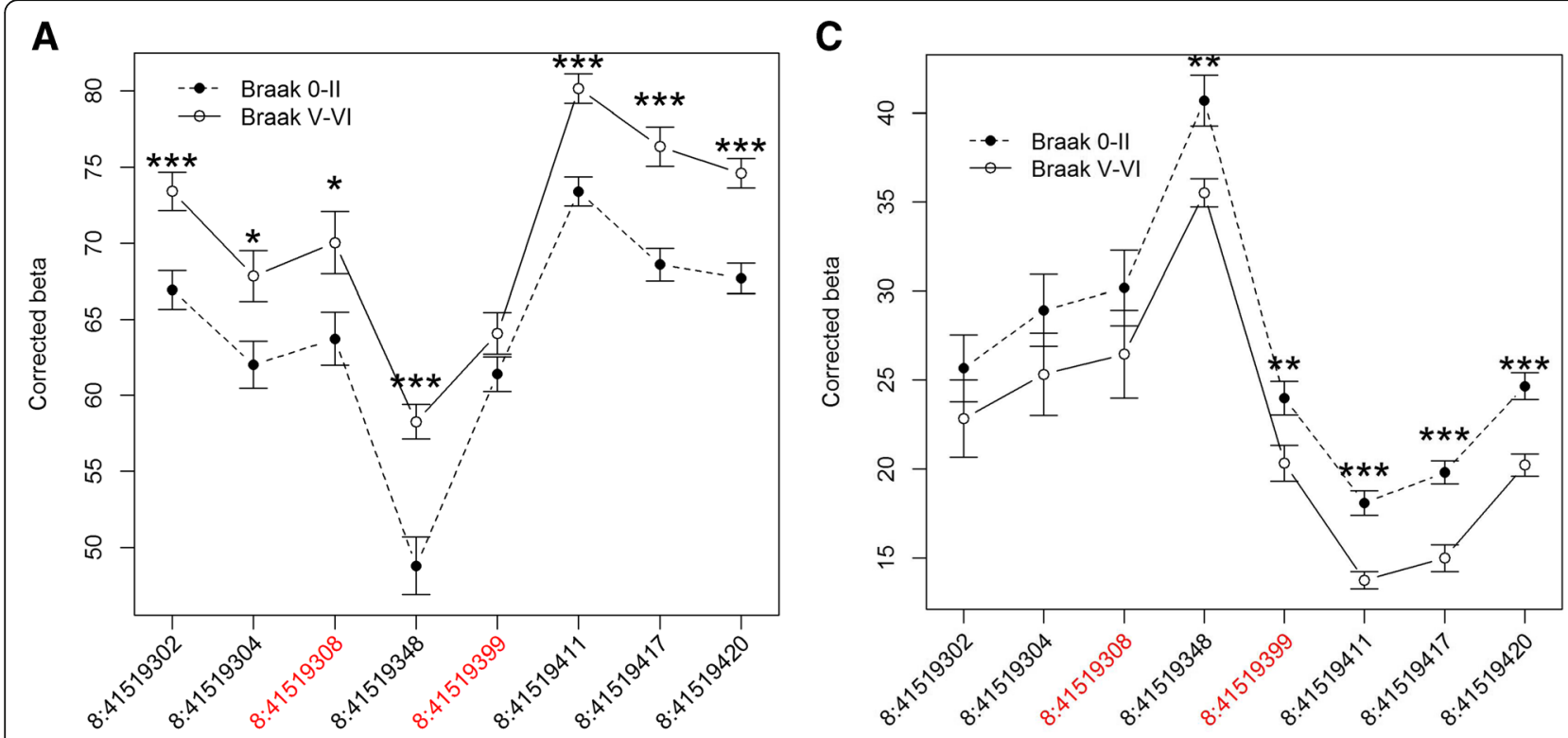

B

D
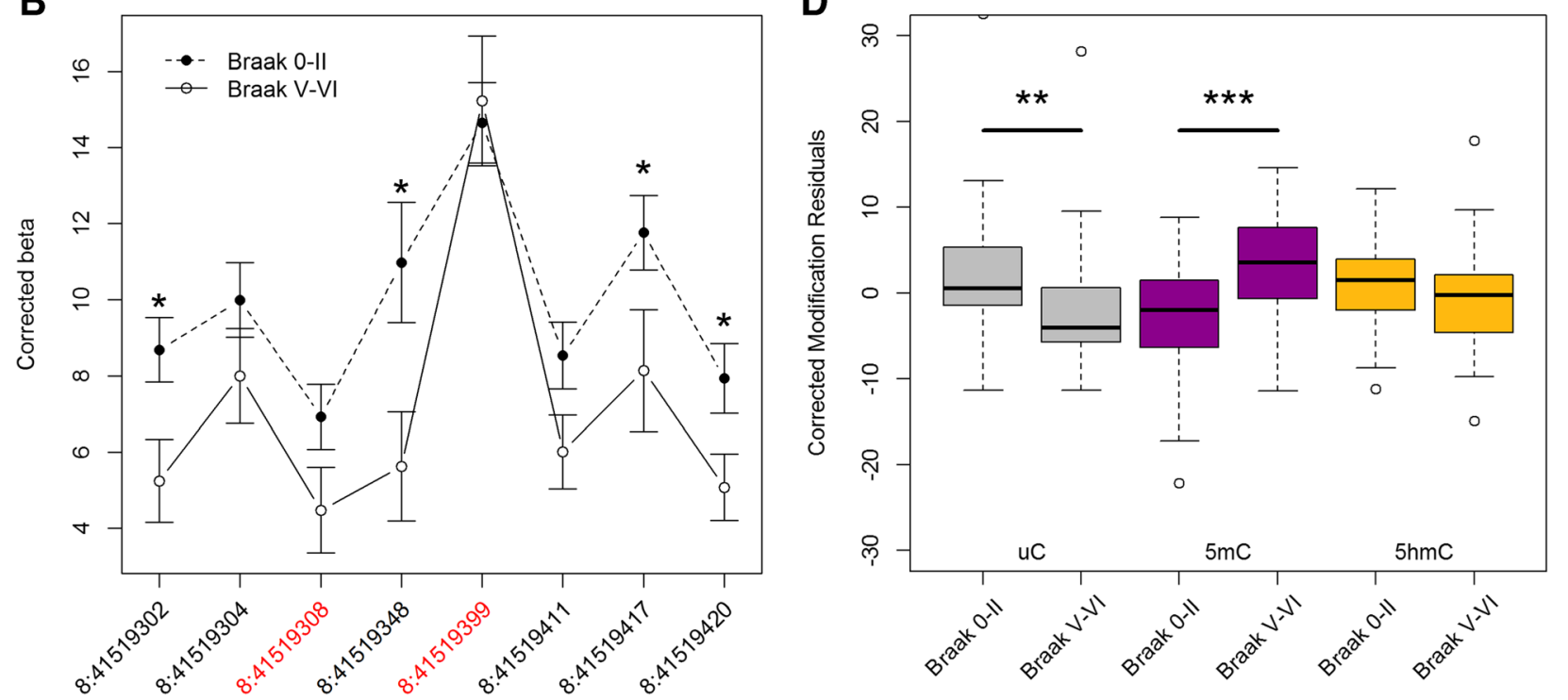

Fig. 5 Sites in ANK1 are characterized by significant DNA hypermethylation and hypohydroxymethylation in AD across an extended region. Using BS and OxBS pyrosequencing, we determined a 5mC, b 5hmC, and $\mathbf{c}$ uC levels in the EC in Braak V-VI samples compared to Braak 0-II in "validation cohort 2." We assayed a 118-bp region containing cg05066959 (chr8:41519308) and cg1182378 (chr8:41519399). We demonstrated significant $(P<0.05)$ neuropathology-associated hypermethylation at seven of the eight CpG sites, significant hypohydroxymethylation at four of the eight CpG sites, and significantly decreased $\mathrm{UC}$ at five of the eight sites. $\mathbf{d}$ Global analysis of all sites within the 118 bp amplicon region highlighted a significant decrease in $\mathrm{UC}$ levels $\left(P=6.72 \times 10^{-3}\right)$ and increase in $5 \mathrm{mC}\left(P=1.23 \times 10^{-5}\right)$ and a trend towards a decrease in $5 \mathrm{hmC}$ $(P=0.058)$ in individuals with Braak $V-V I$, compared to Braak 0-II. Data is represented as mean $\left( \pm\right.$ SEM) Key: ${ }^{*} P<0.05$, ${ }^{* *} P<0.01,{ }^{* *} P<0.005$

silencing, recent studies show this relationship is context dependent [30]. The DMR we identified in $A N K 1$ is within the gene body (exon 42 of the longest isoform), and a number of studies have reported a positive correlation between gene body methylation and expression [31].

\section{Conclusions}

Our study represents, to our knowledge, the first systematic study of $5 \mathrm{mC}$ and $5 \mathrm{hmC}$ in $\mathrm{AD}$, utilizing independent study cohorts and two independent technologies (OxBS-Illumina 450K array and OxBS-pyrosequencing). Two previous studies of DNA hydroxymethylation in $\mathrm{AD}$ have been recently published. The first used a selective chemical labeling technique to enrich for $5 \mathrm{hmC}$, and then sequenced the captured libraries from a small cohort of 30 individuals with either $\mathrm{AD}$, mild cognitive impairment (MCI), or no dementia [32]. The study highlighted a 
number of $\mathrm{AD}$-associated DHPs and demonstrated that gene body DNA hydroxymethylation was positively correlated with cis-acting gene expression. One caveat of that study was that the analysis was unable to discriminate between $5 \mathrm{hmC}$ and $5 \mathrm{mC}$ because of the low sequencing resolution. The second study, by Ellison and colleagues, utilized reduced representation hydroxymethylation profiling (RRHP) to analyze $5 \mathrm{hmC}$ levels in AD hippocampus [33]. Although this study assessed DNA hydroxymethylation at more than two million sites, the study was limited to just three AD cases and two age-matched control subjects. In our study, the overlap between total modifications (BS data) identified across our sample cohorts and those identified by Lunnon et al. [7], despite the use of independent study cohorts, suggests that our study was adequately powered to detect robust $\mathrm{AD}$-associated differences that can be replicated in other studies. Our analyses from multiple independent cohorts provide further evidence for a role for DNA hypermethylation, coupled with hypohydroxymethylation and decreased $\mathrm{uC}$, across a region in ANK1 in AD-associated neuropathology. It indicates that previous estimates of hypermethylation in ANK1 were underestimates as it is potentially confounded by this hypohydroxymethylation. As both $5 \mathrm{hmC}$ and $\mathrm{uC}$ are lower in disease, this suggests the loss of active DNA demethylation in $\mathrm{AD}$. One limitation of our study is that we are unable to determine the functional effects of the disease-associated epigenetic changes we identified and cannot distinguish whether these are causal or secondary to the disease process. Looking to the future, mechanistic experiments using epigenetic editing in induced pluripotent stem cell (iPSC) models are warranted to explore the changes we identified in WNT5B, ANK1, ARID5B, $F B X L 16, A L L C$, and JAG2. These would use, for example, a modified version of the CRISPR-Cas9 technology to alter DNA methylation at these specific loci, and examine their effect on cell function [34].

\section{Methods}

\section{Subjects and samples}

For BS and OxBS Illumina 450K profiling ("discovery cohort"), we used EC brain samples collected from 96 individuals archived in the MRC London Neurodegenerative Disease Brain Bank (https://www.kcl.ac.uk/ioppn/depts/ bcn/our-research/neurodegeneration/brain-bank.aspx).

These samples were not utilized in previously published AD EWAS publications [7, 35, 36]. Our first "validation cohort" consisted of previously published BS EWAS data generated in 104 EC brain samples [7]. Our second "validation cohort" (for pyrosequencing) consisted of 96 EC brain archived in the Thomas Willis Oxford Brain Collection (https://oxfordbrc.nihr.ac.uk/research-themes-overview/ oxford-biorepository/thomas-willis-brain-collection/) [25].
For the "discovery cohort" and "validation cohort 1," individuals had varying degrees of AD pathology (Braak Stage $0-\mathrm{VI})$ and this was used as a measure of degree of pathology in the analyses. For "validation cohort 2," no Braak stage III-IV samples were available so individuals in this cohort were classified as control (Braak 0-II) or cases (Braak $\mathrm{V}-\mathrm{VI}$ ) for analyses. All samples were dissected by trained specialists, snap-frozen, and stored at $-80^{\circ} \mathrm{C}$. Further information about all samples is provided in Table 1. For the "discovery cohort" and "validation cohort 2," genomic DNA was isolated from $\sim 100 \mathrm{mg}$ of each dissected brain region using a standard phenol-chloroform extraction method and tested for degradation and purity prior to analysis.

\section{Methylomic and hydroxymethylomic profiling}

One microgram of DNA from each sample ("discovery cohort" and "validation cohort 2") was treated with sodium BS and OxBS in parallel using the true-methyl CEGX 96 kit (CEGX, Cambridge, UK) according to the manufacturer's standard protocol. Briefly, DNA samples were split, with half being oxidized (OxBS) and the remainder (BS) going through a mock oxidization step, before all being BS treated. All "discovery cohort" samples were then processed using the Illumina Infinium Human Methylation $450 \mathrm{~K}$ Microarray ( $450 \mathrm{~K}$ array) (Illumina Inc., CA, USA) according to the manufacturer's instructions, with minor amendments and quantified using an Illumina HiScan System (Illumina, CA, USA). "Validation cohort 2" samples were used for pyrosequencing (see the "Targeted replication using bisulfite pyrosequencing" section).

For the $450 \mathrm{~K}$ array experiments, matched OxBS- and BS-treated DNA from the same sample were run together on the same $450 \mathrm{~K}$ array. Samples were assigned a unique code for the purpose of the experiment, were randomized in their OxBS and BS pairs with respect to sex and disease status to avoid batch effects, and processed in batches of 12. Illumina Genome Studio software was used to extract the raw signal intensities of each probe (without background correction or normalization).

\section{Data analysis}

All computations and statistical analyses were performed using R 3.3.2 [37] and Bioconductor 3.5 [38]. Signal intensities were imported into $\mathrm{R}$ using the methylumi package [39] as a methylumi object. Initial quality control (QC) checks were performed using functions in the methylumi package to assess concordance between reported and genotyped gender. Probes with common (minor allele frequency $(\mathrm{MAF})>5 \%$ ) SNPs in the CG or single base extension position or probes that are nonspecific or mismapped were flagged and discarded from our results [40]. Data was pre-processed in the $R$ package wateRmelon using the dasen function as previously 
described [41]. The pfilter function was used to remove samples where $5 \%$ of sites had a detection $P$ value $>0.05$. Specific sites were also removed if the beadcount was less than three in $5 \%$ of samples or if $1 \%$ of the samples had a detection $P>0.05$ at that position. After these QC steps, 367,480 probes were taken forward for analysis. Array data for each treatment was normalized separately. To calculate the proportion of DNA modifications, we used the maximum likelihood methylation levels (MLML) method described by Qu et al. [42]. We used the MLML function within the MLML2R package [43], which uses combined signals from BS and OxBS arrays as an input and returns the estimated proportion of $\mathrm{uC}, 5 \mathrm{mc}$, and $5 \mathrm{hmc}$ for each CpG site. In previous studies, we observed that the naive subtraction of the OxBS signal from the BS signal results in a negative value for $5 \mathrm{hmC}$ for a proportion of $\mathrm{CpG}$ sites, likely resulting from technical variance inherent in the Illumina array protocol [11]. When we assessed the distribution of BS-oxBS values, we revealed an accumulation of negative values mainly when the BS $450 \mathrm{~K}$ array beta value at that given site is $<0.1$. Therefore, only probes with a mean beta value $>0.1$ on both the BS and oxBS $450 \mathrm{~K}$ arrays were included in the MLML method ( $N=$ 355,360 probes). Finally, for $5 \mathrm{hmC}$, we only used sites in our analysis when $5 \mathrm{hmC}$ was present in more than half of the sample population ( $N=219,435$ probes).

For the identification of DMPs, DHPs, and DUPs specifically altered with respect to neuropathological measures of $\mathrm{AD}$, we performed a quantitative analysis where samples were analyzed with respect to Braak stage using a linear regression model, and age, sex and neuron/glia proportions were used as co-variates within the analysis. Neuron/glia proportions were calculated using the CETS package in $\mathrm{R}$ [44]. We performed four linear regression models looking for an association with Braak stage, while controlling for the three co-variates. The first model looked for an association with total DNA modifications (BS data), which was just used to demonstrate consistent effect sizes compared to another previously published EWAS in EC (Fig. 1), and for this analysis, we used the 91 BS samples that passed QC (Table 1). The other models looked for an association with $5 \mathrm{mC}$ (OxBS data), $5 \mathrm{hmC}$ (BS - OxBS data), or uC (1-BS data) using the output from the MLML method, and in these analyses, we used the 85 samples where the BS and OxBS data both passed QC (Table 1). For each model, Q-Q plots were assessed to check for $P$ value inflation (see Additional file 2: Figure S1). Probes were ranked by $P$ value, and loci were deemed to be significant if the $P$ value was smaller than the multiple testing threshold for $450 \mathrm{~K}$ array data ("experiment-wide significance"), which was recently reported to be $2.4 \times 10^{-7}$ [45]. The symbol " $\Delta$ " in this manuscript refers to the percentage methylation difference between Braak 0 and Braak VI individuals.

\section{Differentially methylated regions}

To identify DMRs, DHRs, and DURs, we identified spatially correlated $P$ values within our data using the Python module comb-p [17]. This method works by grouping spatially correlated CpGs within a 500-bp sliding window with a significance threshold of $P<0.01$ into putative regions with a corresponding (uncorrected) regional $P$ value. For each putative region, the module then assigns a Sidak-corrected $P$ value to account for multiple testing. DMRs, DHRs, and DURs were considered significant if the Sidak-corrected $P$ value was $<0.05$.

\section{Targeted replication using bisulfite pyrosequencing}

$\mathrm{BS}$ and OxBS pyrosequencing was used to quantify $5 \mathrm{mC}$, $5 \mathrm{hmC}$, and $\mathrm{uC}$ across eight individual ANK1 CpG sites, including cg05066959 and cg11823178, spanning from 41519302 to 41519420 within chromosome 8 (hg19). A single amplicon (246 bp) was amplified using primers designed using the PyroMark Assay Design software 2.0 (Qiagen, UK) as previously described [7], and sequenced using two sequencing primers to maximize coverage across eight CpG sites within a 118-bp region. DNA methylation was quantified in "validation cohort 2" using the PyroMark Q24 system (Qiagen, UK) following the manufacturer's standard instructions and the Pyro Q24 CpG 2.0.6 software. 5hmC values were calculated by the subtraction of the oxBS signal from the $\mathrm{BS}$ signal, $\mathrm{uC}$ values were calculated by subtracting the BS signals from 100 , and $5 \mathrm{mC}$ values were equal to the OxBS signal. Data was adjusted for the effects of age and sex. For this sample cohort, we had no samples with middle stage $\mathrm{AD}$ (Braak III-IV). In this instance, rather than performing a linear regression analysis across Braak stages, we instead compared control samples (Braak scores $0-\mathrm{II})$ to $\mathrm{AD}$ samples (Braak scores $\mathrm{V}-\mathrm{VI}$ ) for $5 \mathrm{mC}$, $5 \mathrm{hmC}$, and $\mathrm{uC}$ levels at eight individual $\mathrm{CpG}$ sites and averaged across the 118-bp amplicon.

\section{Additional files}

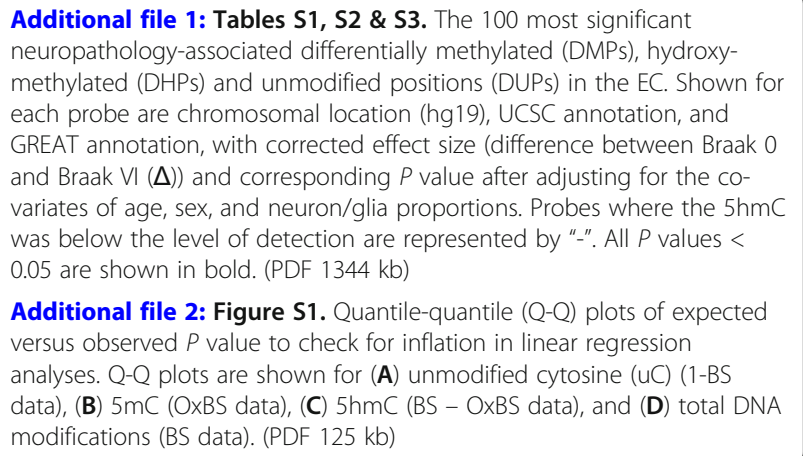

Additional file 2: Figure S1. Quantile-quantile (Q-Q) plots of expected versus observed $P$ value to check for inflation in linear regression analyses. Q-Q plots are shown for (A) unmodified cytosine (UC) (1-BS data), (B) $5 \mathrm{mC}$ (OxBS data), (C) 5hmC (BS - OxBS data), and (D) total DNA modifications (BS data). (PDF $125 \mathrm{~kb}$ )

\section{Abbreviations}

450K array: Illumina 450K methylation array; 5hmC: 5-Hydroxymethylctosine; 5mC: 5-Methylcytosine; AD: Alzheimer's disease; A 3 : Amyloid beta; 
BS: Bisulfite; CGI: CpG island; DHP: Differentially hydroxymethylated position; DHR: Differentially hydroxymethylated region; DMP: Differentially methylated position; DMR: Differentially methylated region; DUP: Differentially unmodified cytosine position; DUR: Differentially unmodified cytosine region; EC: Entorhinal cortex; EWAS: Epigenome-wide association study; GWAS: Genome-wide association study; MAF: Minor allele frequency; NFT: Neurofibrillary tangle; OxBS: Oxidative bisulfite; QC: Quality control; SNP: Single nucleotide polymorphism; uC: Unmodified cytosine

\section{Acknowledgements}

We thank the Brains for Dementia Research (BDR) Initiative (Alzheimer Brain Bank UK) and the donors and families who made this research possible. The Oxford Brain Bank is supported in part by the NIHR Oxford Biomedical Research Centre based at Oxford University Hospitals NHS Trust and University of Oxford

\section{Funding}

This work was funded by an Alzheimer's Association US New Investigator Research Grant (NIRG-14-320878) to KL, a PhD studentship from BRACE (Bristol Research into Alzheimer's and Care of the Elderly) to $\mathrm{KL}$, a major project grant from the Alzheimer's Society UK (AS-PG-14-038) to KL, a pilot project grant to KL from Alzheimer's Research UK (ARUK-PPG2017B-021), an Equipment Grant from Alzheimer's Research UK (ART-EG2010A-2) to JM and project grant funds from the Medical Research Council (MRC) (MR/N027973/ 1) to $\mathrm{KL}$ as part of the Joint Programme-Neurodegenerative Disease Research (JPND) funded consortium project "Targeting epigenetic dysregulation in the brainstem in Alzheimer's Disease (EPI-AD)" to DLvdH from the European Union's Horizon 2020 research and innovation programme under Grant Agreement No. 643417. More generally the EPI-AD project is supported through the following funding organizations under the aegis of JPND—http://www.jpnd.eu: The Netherlands Organization for Health Research and Development (ZonMw) [The Netherlands], Medical Research Council [United Kingdom], German Federal ministry of Education and Research (BMBF) [Germany], National Research Fund (FNR) [Luxembourg].

\section{Availability of data and materials}

The 450K array dataset generated and analyzed during the current study is openly available from GEO under the accession number GSE105109.

\section{Authors' contributions}

$A S$ and JB conducted the laboratory experiments. AS, RS, EP, EH, and JR undertook the data analysis and bioinformatics. CT, SAS, and CS provided samples for analysis. $\mathrm{KL}, \mathrm{DLvdH}$, and JM conceived the project and $\mathrm{KL}$ supervised the project. AS and KL drafted the manuscript. All authors read and approved the final submission.

\section{Ethics approval and consent to participate}

Ethical approval for the study was provided by the NHS South East London REC 3.

\section{Consent for publication}

Not applicable.

\section{Competing interests}

The authors declare that they have no competing interests.

\section{Publisher's Note}

Springer Nature remains neutral with regard to jurisdictional claims in published maps and institutional affiliations.

\footnotetext{
Author details

${ }^{1}$ College of Medicine and Health, University of Exeter Medical School, Exeter University, RILD Building Level 4, Royal Devon and Exeter Hospital, Barrack Rd, Exeter EX2 5DW, UK. ²Department of Psychiatry and Neuropsychology, School for Mental Health and Neuroscience, Maastricht University Medical Centre, Maastricht, The Netherlands. ${ }^{3}$ Institute of Psychiatry, King's College London, London, UK. ${ }^{4}$ Nuffield Department of Clinical Neurosciences, University of Oxford, Oxford, UK. ${ }^{5}$ Laboratory of Translational Neuroscience, Division of Molecular Psychiatry, Department of Psychiatry, Psychosomatics and Psychotherapy, University of Würzburg, Würzburg, Germany.
}

Received: 15 November 2018 Accepted: 18 February 2019

Published online: 21 March 2019

\section{References}

1. Prince $M$, et al. World Alzheimer Report 2015 - the global impact of dementia, an analysis of prevalence, incidence, cost and trends. Alzheimers Dis Int. 2015:1-87. https://www.alz.co.uk/research/ WorldAlzheimerReport2015.pdf.

2. Serrano-Pozo A, et al. Neuropathological alterations in Alzheimer disease. Cold Spring Harb Perspect Med. 2011;1(1):a006189.

3. Association, A.s. Alzheimer's disease facts and figures 2016. Alzheimers Dement. 2016;12(4):1-84.

4. Lambert JC, et al. Meta-analysis of 74,046 individuals identifies 11 new susceptibility loci for Alzheimer's disease. Nat Genet. 2013;45(12):1452-8.

5. Lunnon K, Mill J. Epigenetic studies in Alzheimer's disease: current findings, caveats, and considerations for future studies. Am J Med Genet B Neuropsychiatr Genet. 2013;162B(8):789-99.

6. De Jager $\mathrm{PL}$, et al. Alzheimer's disease: early alterations in brain DNA methylation at ANK1, BIN1, RHBDF2 and other loci. Nat Neurosci. 2014;17(9): 1156-63.

7. Lunnon K, et al. Methylomic profiling implicates cortical deregulation of ANK1 in Alzheimer's disease. Nat Neurosci. 2014;17(9):1164-70.

8. Spiers $\mathrm{H}$, et al. 5-hydroxymethylcytosine is highly dynamic across human fetal brain development. BMC Genomics. 2017;18(1):738.

9. Munzel M, et al. Quantification of the sixth DNA base hydroxymethylcytosine in the brain. Angew Chem Int Ed Engl. 2010;49(31):5375-7.

10. Kriaucionis $\mathrm{S}$, Heintz N. The nuclear DNA base 5-hydroxymethylcytosine is present in Purkinje neurons and the brain. Science. 2009;324(5929):929-30.

11. Lunnon K, et al. Variation in 5-hydroxymethylcytosine across human cortex and cerebellum. Genome Biol. 2016;17:27.

12. Braak H, Braak E. Neuropathological stageing of Alzheimer-related changes. Acta Neuropathol. 1991;82(4):239-59.

13. Caricasole A, et al. Induction of Dickkopf-1, a negative modulator of the Wht pathway, is associated with neuronal degeneration in Alzheimer's brain. J Neurosci. 2004;24(26):6021-7.

14. Toledo EM, Inestrosa NC. Activation of Wnt signaling by lithium and rosiglitazone reduced spatial memory impairment and neurodegeneration in brains of an APPswe/PSEN1DeltaE9 mouse model of Alzheimer's disease. Mol Psychiatry. 2010;15(3):272-85 228

15. Riise J, et al. Aberrant Wnt signaling pathway in medial temporal lobe structures of Alzheimer's disease. J Neural Transm (Vienna). 2015;122(9): 1303-18.

16. Slieker RC, et al. Identification and systematic annotation of tissue-specific differentially methylated regions using the Illumina 450k array. Epigenetics Chromatin. 2013;6(1):26.

17. Pedersen BS, et al. Comb-p: software for combining, analyzing, grouping and correcting spatially correlated P-values. Bioinformatics. 2012;28(22):2986-8.

18. Baba A, et al. PKA-dependent regulation of the histone lysine demethylase complex PHF2-ARID5B. Nat Cell Biol. 2011;13(6):668-75.

19. Hollingworth $P$, et al. Common variants at ABCA7, MS4A6A/MS4A4E, EPHA1, CD33 and CD2AP are associated with Alzheimer's disease. Nat Genet. 2011; 43(5):429-35.

20. Naj AC, et al. Common variants at MS4A4/MS4A6E, CD2AP, CD33 and EPHA1 are associated with late-onset Alzheimer's disease. Nat Genet. 2011; 43(5):436-41.

21. Carrasquillo MM, et al. Replication of EPHA1 and CD33 associations with late-onset Alzheimer's disease: a multi-centre case-control study. Mol Neurodegener. 2011;6(1):54.

22. Qin $\mathrm{H}$, et al. Inhibition of the JAK/STAT pathway protects against alphasynuclein-induced neuroinflammation and dopaminergic neurodegeneration. J Neurosci. 2016;36(18):5144-59.

23. Orre $M$, et al. Isolation of glia from Alzheimer's mice reveals inflammation and dysfunction. Neurobiol Aging. 2014;35(12):2746-60.

24. He W, et al. Beta-site amyloid precursor protein cleaving enzyme 1(BACE1) regulates Notch signaling by controlling the cleavage of Jagged 1 (Jag1) and Jagged 2 (Jag2) proteins. J Biol Chem. 2014;289(30):20630-7.

25. Esiri MM. Brain banks: the Oxford experience. J Neural Transm Suppl. 1993; 39:25-30.

26. Mastroeni D, et al. ANK1 is up-regulated in laser captured microglia in Alzheimer's brain; the importance of addressing cellular heterogeneity. PLoS One. 2017;12(7):e0177814. 
27. Smith AR, et al. A cross-brain-regions study of ANK1 DNA methylation in different neurodegenerative diseases. Neurobiol Aging. 2019;74:70-6.

28. Voronin DA, Kiseleva EV. Functional role of proteins containing ankyrin repeats. Cell Tissue Biol. 2008:2(1):1-12.

29. Gasparoni G, et al. DNA methylation analysis on purified neurons and glia dissects age and Alzheimer's disease-specific changes in the human cortex. Epigenetics Chromatin. 2018;11(1):41.

30. Varley KE, et al. Dynamic DNA methylation across diverse human cell lines and tissues. Genome Res. 2013;23(3):555-67.

31. Yang $X$, et al. Gene body methylation can alter gene expression and is a therapeutic target in cancer. Cancer Cell. 2014;26(4):577-90.

32. Zhao J, et al. A genome-wide profiling of brain DNA hydroxymethylation in Alzheimer's disease. Alzheimers Dement. 2017;13(6):674-88.

33. Ellison EM, Bradley-Whitman MA, Lovell MA. Single-base resolution mapping of 5-hydroxymethylcytosine modifications in hippocampus of Alzheimer's disease subjects. J Mol Neurosci. 2017;63(2):185-97.

34. Imm J, et al. Using induced pluripotent stem cells to explore genetic and epigenetic variation associated with Alzheimer's disease. Epigenomics. 2017; 9(11):1455-68.

35. Smith $\mathrm{RG}$, et al. Elevated DNA methylation across a 48-kb region spanning the HOXA gene cluster is associated with Alzheimer's disease neuropathology. Alzheimers Dement. 2018:14(12):1580-8.

36. Smith AR, et al. Increased DNA methylation near TREM2 is consistently seen in the superior temporal gyrus in Alzheimer's disease brain. Neurobiol Aging. 2016;47:35-40.

37. R Development Core Team. R: a language and environment for statistical computing. Vienna: R Foundation for statistical computing; 2012. p. 2012.

38. Gentleman RC, et al. Bioconductor: open software development for computational biology and bioinformatics. Genome Biol. 2004;5(10):R80.

39. Davis, S., et al., Methylumi: handle Illumina methylation data 2012.. R package version 2.2.0 2012

40. Chen YA, et al. Discovery of cross-reactive probes and polymorphic CpGs in the Illumina Infinium HumanMethylation450 microarray. Epigenetics. 2013; 8(2):203-9.

41. Pidsley $\mathrm{R}$, et al. A data-driven approach to preprocessing Illumina 450K methylation array data. BMC Genomics. 2013;14:293.

42. Qu J, et al. MLML: consistent simultaneous estimates of DNA methylation and hydroxymethylation. Bioinformatics. 2013;29(20):2645-6.

43. Samara K, et al. MLML2R: maximum likelihood estimation of DNA methylation and hydroxymethylation proportions. R package version 0.3.0. 2018. https://cran.r-project.org/package=MLML2R.

44. Guintivano J, Aryee M, Kaminsky Z. A cell epigenotype specific model for the correction of brain cellular heterogeneity bias and its application to age, brain region and major depression. Epigenetics. 2013;8(3):290-302.

45. Saffari A, et al. Estimation of a significance threshold for epigenome-wide association studies. Genet Epidemiol. 2018:42(1):20-33.

Ready to submit your research? Choose BMC and benefit from:

- fast, convenient online submission

- thorough peer review by experienced researchers in your field

- rapid publication on acceptance

- support for research data, including large and complex data types

- gold Open Access which fosters wider collaboration and increased citations

- maximum visibility for your research: over $100 \mathrm{M}$ website views per year

At $\mathrm{BMC}$, research is always in progress.

Learn more biomedcentral.com/submissions 